\title{
Optimal Placement of Active Bars for Buckling Control in Truss Structures under Bar Failures
}

\section{Tristan Gally ${ }^{1, a^{*}}$, Anja Kuttich ${ }^{1, b}$, Marc E. Pfetsch ${ }^{1, \mathrm{c}}$, Maximilian Schaeffner ${ }^{2, \mathrm{~d}}$ and Stefan Ulbrich ${ }^{1, \mathrm{e}}$}

\author{
${ }^{1}$ TU Darmstadt, Optimization, Dolivostr. 15, 64293 Darmstadt, Germany \\ ${ }^{2}$ TU Darmstadt, System Reliability and Machine Acoustics SAM, Magdalenenstr. 4, 64289 \\ Darmstadt, Germany \\ agally@mathematik.tu-darmstadt.de, bkuttich@mathematik.tu-darmstadt.de, \\ cpfetsch@mathematik.tu-darmstadt.de, ${ }^{\mathrm{d}}$ schaeffner@sam.tu-darmstadt.de, \\ eulbrich@mathematik.tu-darmstadt.de
}

Keywords: truss topology design, active buckling control, resilience, actuator placement

\begin{abstract}
Buckling of slender bars subject to axial compressive loads represents a critical design constraint for light-weight truss structures. Active buckling control by actuators provides a possibility to increase the maximum bearable axial load of individual bars and, thus, to stabilize the truss structure.

For reasons of cost, it is in general not economically viable to use such actuators in each bar of the truss structure. Hence, it is an important practical question where to place these active bars. Optimized structures, especially when coupled with active elements to further decrease the number of necessary bars, however, lead to designs, which, while cost-efficient, are especially prone to bar damages, caused, e.g., by material failures. Therefore, this paper presents a mathematical optimization approach to optimally place active bars for buckling control in a way that secures both buckling and general stability constraints even after failure of any combination of a certain number of bars. This allows us to increase the resilience of the system and guarantee stable behavior even in case of failures.
\end{abstract}

\section{Introduction}

An important task in mechanical engineering is the design of truss structures. The goal is to build structures which are light-weight but at the same time stable when exposed to external loads. In most of the previous works on optimal truss topology design, the stability is measured by the so-called compliance, the potential energy stored in the deformed truss, see, e.g., Bendsøe and Sigmund [1], whereas other stability qualities such as buckling are ignored. In practice, however, the avoidance of buckling is actually an important requirement. This is rarely considered in optimization approaches, and if so, only on a global level, as in Ben-Tal et al. [2].

Apart from increasing the diameter of bars which are particularly prone to buckling because of the topology of the truss, another way to avoid buckling is active buckling control via piezoelectric stack actuators. These active elements can increase the critical buckling load of a given bar and therefore its maximum bearable axial load. Since these active bars may be significantly more expensive than passive ones, however, it may not always be an option to integrate this technology into all bars of a truss. Thus, it is necessary to determine the correct bars to optimally place the active elements within the truss to increase the stability of the whole truss even with a small number of active bars.

Besides stability conditions that prevent the buckling of bars, also the control of uncertainty is an important task in truss topology design. One particular problem of optimizing truss structures is that the optimization will lead to designs which are only stable for the given loads, while even small disturbances may lead to an arbitrarily large compliance or failure of the buckling constraints. For this reason, truss topology design is naturally suited to the idea of robust optimization [3], optimizing the worst-case of the compliance over all loads in a given uncertainty set. Even when designing for this larger set of potential loads using robust optimization, however, not all uncertain effects can be ruled out. For example wear or manufacturing errors could still reduce the stability of bars or even cause 
them to outright fail. Therefore, the truss should be designed in a way that it still maintains its stability even after removal of a given number of arbitrary bars, thereby designing resilient trusses.

While robust optimization based approaches to truss topology design have been heavily pursued over the last decades, complete bar failures have only been investigated very recently, apart from some early work of Sun et al. [4], which only considers a small, predetermined set of failure scenarios. Jansen et al. [5] and Zhou and Fleury [6] work on continuous topology optimization problems, distributing material among a space of "pixels" under the constraint that even after erasing all material within a ball or cube of given size, the structure should still be able to sustain its load. Mohr et al. [7] consider a ground structure approach, but in their model not only single bars can fail, but instead $n$ complete trusses have to be built from a given ground structure such that either a single one of them or each set of $n-1$ trusses together can withstand the given load after complete failure of all the remaining trusses. The work most similar to ours is that of Kanno [8], who also considers arbitrary failures of at most $k$ bars, but only considers displacement constraints.

In the following, we will introduce the technology for active buckling control, before extending a basic mixed-integer semidefinite programming (MISDP) model for truss topology design by buckling constraints, optimal placement of active bars for buckling control and to the case of potential bar failures. Afterwards, we will show numerical results illustrating the potential of the method, before discussing future research directions, in particular related to the scalability of the model to real-world problem sizes.

\section{Buckling Control}

Truss structures that bear and withstand static or time-varying loads are widely used in modern lightweight mechanical and civil structures. The trusses consist of individual bars that are mainly loaded in tension or compression. In case of compressive axial loads, buckling of the individual bars has to be considered as the critical failure mode as it may lead to the collapse of an entire truss structure. Buckling occurs as a stability failure at the compressive critical buckling load $F_{\text {crit }}$ resulting in large lateral deformations of the bar, [9, 10].

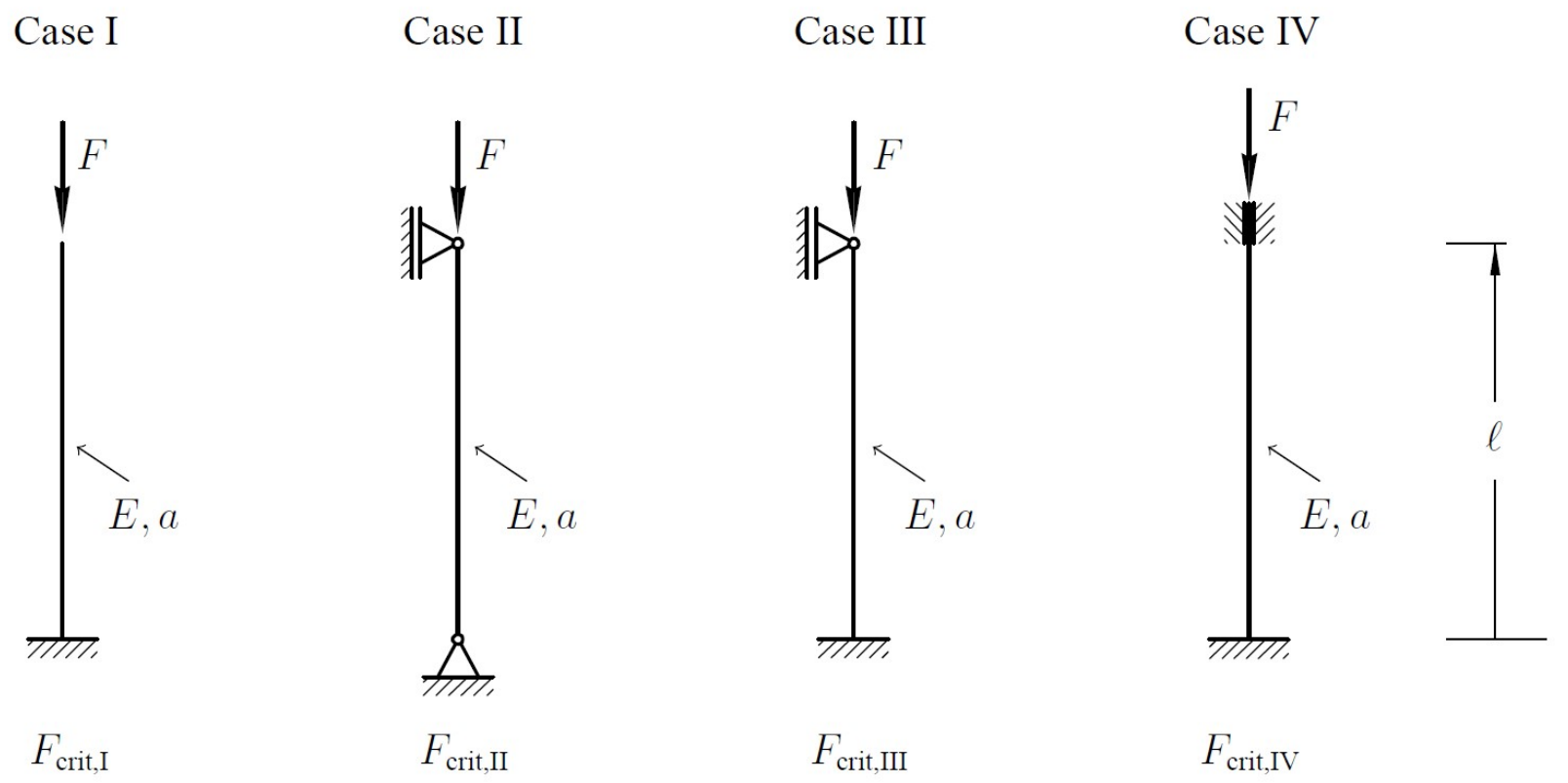

Fig. 1: Euler buckling cases I (fixed-free), II (pinned-pinned), III (fixed-pinned) and IV (fixed-fixed) 
The critical buckling load depends on the boundary conditions of the axially loaded bar. Figure 1 shows the four Euler buckling cases of which Case II with pinned-pinned supports with critical buckling load

$$
F_{\text {crit,II }}=\frac{\pi E a^{2}}{4 \ell^{2}}
$$

is best known. Equation (1) is valid for a bar with circular solid cross-sectional area $a$, length $\ell$ and Young's modulus $E$. The other boundary conditions fixed-free in Case I, fixed-pinned in Case III and fixed-fixed in Case IV lead to critical buckling loads of $F_{\text {crit,I }}=0.25 F_{\text {crit,II }}, F_{\text {crit,III }}=2.046 F_{\text {crit,II }}$ and $F_{\text {crit,IV }}=4 F_{\text {crit,II. }}$. From (1), it can be seen that the critical buckling load of a bar may passively be increased by changing its geometry, i.e. length and cross-sectional area, or the material, i.e. Young's modulus.

Alternative to passively increasing the maximum bearable compressive load of an individual bar, active buckling control may be used. Active buckling control provides a possibility to increase the maximum bearable load of slender bars by the integration of piezoelectric stack actuators in compact piezo-elastic supports at the bar ends, Fig.2(a), [11, 12]. In this particular setup, the circular bar is made of aluminum alloy EN AW-7075 with a length of $400 \mathrm{~mm}$, diameter of $8 \mathrm{~mm}$ and Young's modulus $71.0 \mathrm{kN} / \mathrm{mm}^{2}$. The boundary conditions are close to Euler Case IV with fixed-fixed supports, Fig. 1. The piezo-elastic supports introduce active bending moments that act in arbitrary directions at the bar ends so that buckling and large lateral bar deformations may be prevented.

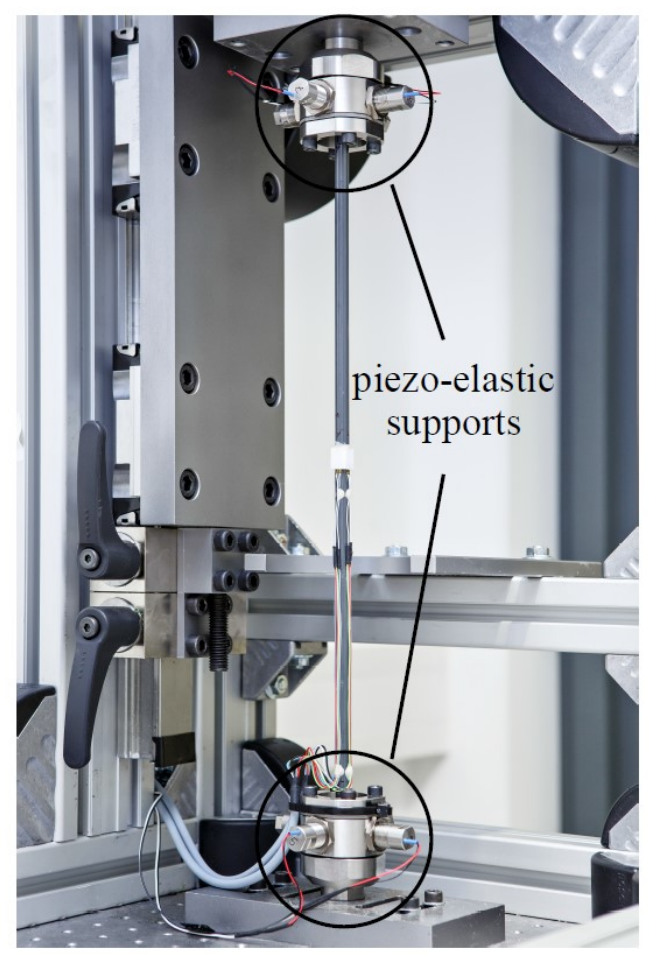

(a)

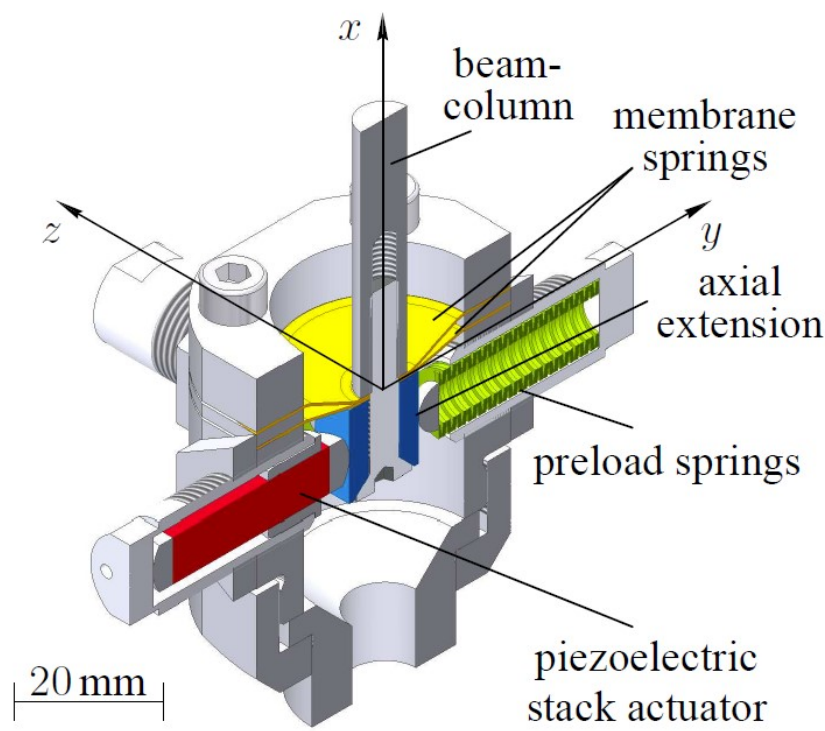

(b)

Fig. 2: (a) active bar with piezo-elastic supports for buckling control (photo: Fraunhofer LBF, Darmstadt, Germany), (b) sectional view of the piezo-elastic support

A sectional view of the piezo-elastic support is shown in Fig.2(b). The central element of the support are two concave-shaped elastic membrane springs that bear the axial and lateral loads and allow rotations in any plane perpendicular to the $x$-axis. Two piezoelectric stack actuators that are mechanically prestressed by allocated preload springs are arranged orthogonal to each other and orthogonal to the beam-column's $x$-axis, acting in $y$ - and $z$-direction. They exert lateral forces to the bar's axial 
extension that works as a cantilever beam end beyond the elastic membrane springs for each support. This way, the active lateral forces provided by the piezoelectric stack actuators act in arbitrary directions orthogonal to the beam-column's longitudinal $x$-axis. A gain-scheduled $H_{\infty}$ buckling control is used to stabilize an individual bar in arbitrary lateral direction, [12]. With the proposed control it is possible to increase the maximum bearable load of the active bar in an experimental investigation by $19 \%$ or $625 \mathrm{~N}$ for quasi-static axial loads compared to the passive bar.

The active bar from Fig.2(a) can be used to replace highly compressively loaded passive bars in truss structures that would otherwise need to have a larger cross-sectional area. In order to determine an optimal placement of the active bars in a truss structure, mathematical optimization is used, as described in the following sections.

\section{An MISDP Model for Optimal Actuator Placement under Bar Failures}

In this section, we develop a mixed integer-semidefinite program to optimize the topology of truss structures and to optimally place the active bars presented in the last section such that the truss is able to withstand a given set of loads even in the presence of bar failures. We build upon the basic semidefinite model for truss topology design of Ben-Tal and Nemirovski where a fixed ground structure is given and the goal is to find optimal cross-sectional areas which minimize a given quality criterion [13]. The ground structure consists of a set of nodes $\mathcal{V}:=\left\{v_{1}, \ldots, v_{n}\right\} \subseteq \mathbb{R}^{d}$ and a set of $m$ potential bars $\mathcal{E} \subseteq \mathcal{V} \times \mathcal{V}$. The set of nodes is further divided into $n_{f}$ free nodes in $\mathcal{V}_{f} \subseteq \mathcal{V}$, which are movable, and the remaining fixed nodes. Given an external load $f \in \mathbb{R}^{d_{f}}$ with $d_{f}=d \cdot n_{f}$ or set of loads $\mathcal{S}:=\left\{f_{1} \ldots, f_{s}\right\}$, the goal is to find optimal cross-sectional areas $a_{e}$ for all bars $e \in \mathcal{E}$ such that the compliance $f^{\top} u / 2$ of the resulting truss is less than a given upper bound $C_{\max } \in \mathbb{R}_{+}$for all loads in the scenario-set $\mathcal{S}$. The node displacements $u$ can be determined through the equilibrium condition $A(a) u=f$ with stiffness matrix $A(a)=\sum_{e \in \mathcal{E}} A_{e} \ell_{e} a_{e}$ combining the bar stiffness matrices $A_{e}=E / \ell_{e}^{2} \cdot b_{e} b_{e}^{\top}$, where

$$
b_{e}=\left(b_{\left(v_{i}, v_{j}\right)}(k)\right)_{\left\{k \leq n_{f}\right\}}= \begin{cases}\frac{v_{i}-v_{j}}{\left\|v_{j}-v_{i}\right\|_{2}}, & k=i \\ \frac{v_{j}-v_{i}}{\left\|v_{j}-v_{i}\right\|_{2}}, & k=j \\ 0, & \text { otherwise }\end{cases}
$$

with $E$ the Young's modulus of the used material and $\ell_{e}$ the length of bar $e$.

Since in practice it is not always possible to buy or even produce bars of arbitrary thickness, we will follow the approach of Kočvara [14] and Mars [15] and only allow cross-sectional areas in some predetermined set $\mathcal{A}:=\left\{a_{1}, \ldots, a_{k}\right\}$. Therefore, define binary variables $x_{e}^{a}$ such that the crosssectional area of bar $e$ is $a$ if and only if $x_{e}^{a}=1$. Then the stiffness matrix can be rewritten as

$$
A(x)=\sum_{e \in \mathcal{E}} \sum_{a \in \mathcal{A}} A_{e} \ell_{e} a x_{e}^{a},
$$

which leads to the mixed-integer semidefinite program

$$
\begin{aligned}
& \min \sum_{e \in E} \ell_{e} \sum_{a \in \mathcal{A}} a x_{e}^{a} \\
& \text { s.t. } \quad\left(\begin{array}{cc}
2 C_{\max } & f_{s}^{\top} \\
f_{s} & A(x)
\end{array}\right) \succeq 0, \quad \forall f_{s} \in \mathcal{S}, \\
& \sum_{a \in \mathcal{A}} x_{e}^{a} \leq 1, \quad \forall e \in \mathcal{E}, \\
& x_{e}^{a} \in\{0,1\}, \quad \forall e \in \mathcal{E}, a \in \mathcal{A} \text {. }
\end{aligned}
$$


The presented optimization model for truss topology design is a simple model which does not take into account further stability qualities such as buckling. To cover such stability conditions, we extend the model by buckling constraints in the next section.

Buckling Constraints. To prevent a bar $e$ from buckling or to check whether bar $e$ buckles at all, we must first determine the bar force $\left(q_{s}\right)_{e}$ for each scenario $f_{s} \in \mathcal{S}$ and each bar $e \in \mathcal{E}$. This can be done by using the so-called geometry matrix $B=\left(b_{1}, \ldots, b_{m}\right) \in \mathbb{R}^{d_{f} \times m}$ and the equilibrium conditions $B q_{s}=f_{s}$ for each load scenario $f_{s} \in \mathcal{S}$. These uniquely determine the bar forces, as long as $B$ is invertible. This assumption, usually referred to as a statically determined truss, is satisfied if the number of bars is equal to the degrees of freedom of the system, so $m=d_{f}$, and additionally the set of vectors $\left(b_{e}\right)_{e \in \mathcal{E}} \subseteq \mathbb{R}^{d_{f}}$ is linearly independent.

As presented in the previous section, to prevent bars under compression from buckling, we have to guarantee that the bar force $\left(q_{s}\right)_{e}$ of bar $e$ is bounded by the critical buckling force, i.e., $-F_{\text {crit }, e} \leq\left(q_{s}\right)_{e}$ for all load scenarios $f_{s} \in \mathcal{S}$. The critical buckling force based on Euler Case II, Fig. 2, is given by the linear term

$$
F_{\text {crit }, e}(x)=\sum_{a \in \mathcal{A}} \frac{\pi E a^{2}}{4 \ell_{e}^{2}} x_{e}^{a},
$$

compare Equation (1). For bars under tension we get an upper bound of $R_{p ; 0,2} \cdot a$ with proportional limit $R_{p ; 0,2}$. Together this gives us the constraint

$$
-F_{\text {crit }, e}(x) \leq\left(q_{s}\right)_{e} \leq R_{p ; 0,2} \sum_{a \in \mathcal{A}} a x_{e}^{a} \quad \forall f_{s} \in \mathcal{S}, e \in \mathcal{E} .
$$

Note that the bar forces appearing in (6) are not part of the semidefinite model, so we need to introduce them into (4). As long as the geometry matrix $B$ is invertible, this can be done by adding variables $q_{s} \in \mathbb{R}^{m}$ and the equilibrium constraint $B q_{s}=f_{s}$ for each force scenario $f_{s} \in \mathcal{S}$.

To optimally place the active bars described in the last section, we introduce additional binary variables $y_{e}$ with value one if an actuator for buckling control should be placed into bar $e$. Since we only have data for a single diameter, we assume that the increase in the critical buckling force is additive, meaning it is independent of the cross-sectional area. Then we can replace $F_{\text {crit, } e}(x)$ in $(6)$ by

$$
F_{\text {crit }, e}(x, y)=\sum_{a \in \mathcal{A}} \frac{\pi E a^{2}}{4 \ell_{e}^{2}} x_{e}^{a}+\rho y_{e},
$$

with $\rho$ being the absolute additive increase in the critical buckling load through the active bars. Of course, it would also be possible to use a multiplicative term $F_{\text {crit }, e}(x, y)=F_{\text {crit }, e}(x) \cdot(1+\rho y)$ instead of the additive term in (7). Note that the multiplicative term would lead to a nonlinear buckling constraint, although it could be linearized (at the cost of an increased size) by introducing variables $z_{e}=x_{e} y_{e}$, which would still keep the model exact in all integral points. However, since we will later only use bars which are at least as thick as the ones used in the experiments, choosing an additive term based on the experiments is a conservative assumption, which should give a lower bound on the actual increase of the critical buckling force.

Additionally, to enforce that actuators may only be placed in bars which are actually used, we add constraints

$$
y_{e} \leq \sum_{a \in \mathcal{A}} x_{e}^{a}
$$

for all bars $e$. Finally, we may bound the total number of active bars through a constraint

$$
\sum_{e \in \mathcal{E}} y_{e} \leq r
$$

To sum up, for statically determined trusses, adding the equilibrium condition, the buckling condition (7) as well as (8) and (9) yields a mixed-integer semidefinite problem to optimize the topology of truss structures under buckling constraints and to optimally place active bars within the structure. 
Bar Failures. The goal of this section is to extend the model in such a way that both the compliance bound as well as the buckling constraints are not only guaranteed for the original truss, but also after the failure of prespecified sets of bars. For this purpose, let us introduce the set $\mathcal{Z} \subseteq\{0,1\}^{\mathcal{E}}$ of all the possible bar failures which the truss should be able to sustain. Each distinct scenario is then given by a vector $z \in \mathcal{Z} \subseteq\{0,1\}^{\mathcal{E}}$ with $z_{e}=1$ indicating that bar $e$ no longer exists in this scenario. One possible choice for $\mathcal{Z}$, which we will also use for our numerical experiments, is to allow arbitrary failures of up to $k$ bars, so $\mathcal{Z}_{k}:=\left\{z \in\{0,1\}^{\mathcal{E}}:\|z\|_{0} \leq k\right\}$. To consider bar failures, we have to adjust the model presented in the previous subsection in the following way: To correctly bound the compliance of the damaged truss in failure scenario $z^{j}$, we need to adjust the stiffness matrix and replace $A(x)$ by

$$
A\left(x, z^{j}\right)=\sum_{e \in \mathcal{E}} \sum_{a \in \mathcal{A}} A_{e} \ell_{e} a x_{e}^{a}\left(1-z_{e}^{j}\right)
$$

to remove the contribution of all failed bars. For bounding the compliance for all failure scenarios in $\mathcal{Z}$, we then need to replace the single SDP-constraint (4b) by $|\mathcal{Z}|$ SDP-constraints, each replacing the stiffness matrix by the one corresponding to the specific failure scenario. Note that these are still linear in $x$, since the $z$ variables encoding the failure scenario are fixed for each constraint. For the specific choice of $\mathcal{Z}_{k}$, it is in this case also sufficient to only add those constraints corresponding to exactly $k$ failures, since feasibility of the SDP-constraint for $\bar{z}$ implies feasibility for all $z \leq \bar{z}$ by positive semidefiniteness of the $A_{e}$.

With regards to buckling constraints, the geometry matrix $B=B\left(z^{j}\right)$ also needs to be adjusted by removing all columns corresponding to bars failing in scenario $z^{j}$. Therefore, we need to introduce new variables $q_{s}^{j} \in \mathbb{R}^{\mathcal{E}^{j}}$ for the bar forces in scenario $z^{j}$, where $\mathcal{E}^{j}=\left\{e \in \mathcal{E}: z_{e}^{j}=0\right\}$ is the set of intact bars for scenario $z^{j}$. These bar forces can then again be bounded by ranged rows as in (6). Furthermore, it is obvious that the bar forces should still satisfy the constraints

$$
B\left(z^{j}\right) q_{s}^{j}=f_{s}
$$

However, as already indicated, the equilibrium constraint (11) only uniquely determines $q_{s}^{j}$ if $B\left(z^{j}\right)$ is invertible. Depending on the ground structure, this was a valid assumption before, but in the presence of bar failures, not all $B\left(z^{j}\right)$ can be invertible at the same time, since they do not even have the same number of columns unless in all scenarios the exact same number of failures happen (but note that the validity no longer follows automatically for all $z \leq \bar{z}$ ). Therefore, we will additionally need to include the node displacements $u_{s}^{j}$, which are already unique if $A\left(x, z^{j}\right)$ is invertible (which is, e.g., already implied by feasibility of the SDP-constraint and full dimensionality of the set $\mathcal{S}$ ). The node displacements can then be characterized through the equilibrium constraint $A\left(x, z^{j}\right) u_{s}^{j}=f_{s}$. This, however, would make the relaxations non-convex. Therefore, we will instead use the identity

$$
A\left(x, z^{j}\right)=E B\left(z^{j}\right) \operatorname{Diag}\left(\left(a_{e} / \ell_{e}\right)_{e \in \mathcal{E}^{j}}\right) B\left(z^{j}\right)^{\top},
$$

with $a_{e}:=\sum_{a \in \mathcal{A}} a x_{e}^{a}$, which results simply from a comparison of the structure of the stiffness and the geometry matrix. This leads to indicator constraints

$$
x_{e}^{a}=1 \Rightarrow\left(q_{s}^{j}\right)_{e}=\frac{E a}{\ell_{e}} b_{e}^{\top} u_{s}^{j}
$$

which we will later prove to be sufficient for ensuring that $u_{s}^{j}$ is a solution of $A\left(x, z^{j}\right) u_{s}^{j}=f_{s}$ and $q_{s}^{j}$ are the correct bar forces for force $f_{s}$ and failure scenario $z^{j}$. 
Altogether, this gives us the MISDP model

$$
\begin{array}{rlrl}
\min & \sum_{e \in \mathcal{E}} \ell_{e} \sum_{a \in \mathcal{A}} a x_{e}^{a} & \\
\text { s.t. } \quad\left(\begin{array}{cc}
2 C_{\max } & f_{s}^{T} \\
f_{s} & A\left(x, z^{j}\right)
\end{array}\right) & \succeq 0 & & \forall z^{j} \in \mathcal{Z}, f_{s} \in \mathcal{S} \\
\sum_{a \in \mathcal{A}} x_{e}^{a} & \leq 1, & & \forall e \in \mathcal{E}, \\
B\left(z^{j}\right) q_{s}^{j} & =f_{s}, & & \forall z^{j} \in \mathcal{Z}, f_{s} \in \mathcal{S}, \\
-F_{\text {crit }, e}(x, y) \leq\left(q_{s}^{j}\right)_{e} & \leq R_{p ; 0,2} \sum_{a \in \mathcal{A}} a x_{e}^{a}, & & \forall z^{j} \in \mathcal{Z}, f_{s} \in \mathcal{S}, e \in \mathcal{E}^{j}, \\
y_{e} & \leq \sum_{a \in \mathcal{A}} x_{e}^{a}, & & \forall e \in \mathcal{E}, \\
\sum y_{e} & \leq r, & & \\
e \in \mathcal{E} & & \forall z^{j} \in \mathcal{Z}, f_{s} \in \mathcal{S}, e \in \mathcal{E}^{j}, a \in \mathcal{A}, \\
x_{e}^{a}=1 \Rightarrow\left(q_{s}^{j}\right)_{e} & =\frac{E a}{\ell_{e}} b_{e}^{\top} u_{s}^{j}, & & \forall e \in \mathcal{E}, a \in \mathcal{A}, \\
x_{e}^{a} & \in\{0,1\}, & & \forall e \in \mathcal{E}, \\
y_{e} & \in\{0,1\}, & & \forall z^{j} \in \mathcal{Z}, f_{s} \in \mathcal{S}, \\
q_{s}^{j} & \in \mathbb{R}^{\mathcal{E}^{j}}, & & \forall z^{j} \in \mathcal{Z}, f_{s} \in \mathcal{S} . \\
u_{s}^{j} & \in \mathbb{R}^{d_{f}}, &
\end{array}
$$

What remains to be shown for the correctness of the model is that $(14 \mathrm{~h})$ together with the rest of the constraints actually implies that $q_{s}^{j}$ are the correct bar forces for force $f_{s}$ and failure scenario $z^{j}$.

Lemma 1. If $(x, y, q, u)$ is feasible for (14), then

$$
q_{s}^{j}=E \operatorname{Diag}\left(\left(a_{e} / \ell_{e}\right)_{e \in \mathcal{E}^{j}}\right) B\left(z^{j}\right)^{\top} u_{s}^{j},
$$

and $u_{s}^{j}$ is a solution of $A\left(x, z^{j}\right) u_{s}^{j}=f_{s}$, where $a_{e}:=\sum_{a \in \mathcal{A}}$ ax $x_{e}^{a}$. Furthermore, if $A\left(x, z^{j}\right)$ is invertible, then the vector $q_{s}^{j}$ consists of the bar forces for load $f_{s}$ and failure scenario $z^{j}$.

Proof. Let $e \in \mathcal{E}^{j}$. To show that (15) holds, first assume that $\sum_{a \in \mathcal{A}} x_{e}^{a}=1$. Since $x$ is binary, there exists $a \in \mathcal{A}$ such that $x_{e}^{a}=1$. Therefore $\left(q_{s}^{j}\right)_{e}=\frac{E a_{e}}{\ell_{e}} b_{e}^{\top} u_{s}^{j}$ for all $z^{j} \in \mathcal{Z}, f_{s} \in \mathcal{S}$ by (14h). On the other hand, if $\sum_{a \in \mathcal{A}} x_{e}^{a}=0$, then (14e) and (14f) imply $\left(q_{s}^{j}\right)_{e}=0=\frac{E a_{e}}{\ell_{e}} b_{e}^{\top} u_{s}^{j}$, since $a_{e}=0$. Therefore, $\left(q_{s}^{j}\right)_{e}=\frac{E a_{e}}{\ell_{e}} b_{e}^{\top} u_{s}^{j}$ holds for all $e \in \mathcal{E}^{j}$. Combining these rows into a single system of linear equations, we obtain (15). Now from (14d), (15) and since $A\left(x, z^{j}\right)=E B\left(z^{j}\right) \operatorname{Diag}\left(\left(a_{e} / \ell_{e}\right)_{e \in \mathcal{E}^{j}}\right) B\left(z^{j}\right)^{\top}$, we obtain

$$
A\left(x, z^{j}\right) u_{s}^{j}=E B\left(z^{j}\right) \operatorname{Diag}\left(\left(a_{e} / \ell_{e}\right)_{e \in \mathcal{E}^{j}}\right) B\left(z^{j}\right)^{\top} u_{s}^{j}=B\left(z^{j}\right) q_{s}^{j}=f_{s} .
$$

If $A\left(x, z^{j}\right)$ is invertible, the solution of $A\left(x, z^{j}\right) u_{s}^{j}=f$ is unique and characterizes the node displacements for force $f_{s}$ and failure scenario $z^{j}$, which implies that $q_{s}^{j}$ are the bar forces.

\section{Numerical Results}

In this section, we want to present results for Model (14), with the resulting mixed-integer SDPs being solved by modified developer versions of SCIP-SDP 3.1.0 [16] and SCIP 5.0.1 [17] using MOSEK 8.1.0.25 [18] as the SDP-solver on an 8-core Intel i7-4770. Inside SCIP, indicator constraints like (14h) are handled by introducing continuous slack variables, which are fixed to zero once $x_{e}^{a}=1$. 
For comparing the influence of active bars for buckling control and of bar failures, the truss given by the ground structure in Fig. 3 is optimized for the set of failure scenarios $\mathcal{Z}_{k}$ with $k \in\{0,1,2\}$ and with maximum number of active bars $r \in\{0,2\}$. The truss consists of six nodes (of which two are fixed) and twelve potential bars of length between $400 \mathrm{~mm}$ and $895 \mathrm{~mm}$ with cross-sectional areas corresponding to diameters of $8 \mathrm{~mm}, 11 \mathrm{~mm}, 14 \mathrm{~mm}$ and $17 \mathrm{~mm}$. The used material is aluminum with a Young's modulus of $71 \mathrm{kN} / \mathrm{mm}^{2}$ and we enforced two load scenarios with a main force of $0.5 \mathrm{kN}$ in vertical direction in node $F$ with an orthogonal force of $0.1 \mathrm{kN}$ to either the left or the right and additional disturbing forces of $0.05 \mathrm{kN}$ in vertical and $0.01 \mathrm{kN}$ in horizontal direction in all other free nodes. These forces are not enough to guarantee the sufficient condition for invertibility of $A$ given in the last section, nevertheless, we verified that all occurring stiffness matrices are indeed invertible. The compliance was bounded by $10 \mathrm{kNmm}$, and we used a proportional limit of $0.325 \mathrm{kN} / \mathrm{mm}^{2}$ and set the absolute increase of the critical buckling force of an active bar to $0.625 \mathrm{kN}$, the result of the experiments described in the first section. Note that this leads to a higher relative increase, since the buckling force of pinned-pinned bars is smaller by a factor of four compared to that of fixed-fixed bars, which are closer to the ones used in the experiments and simulations.

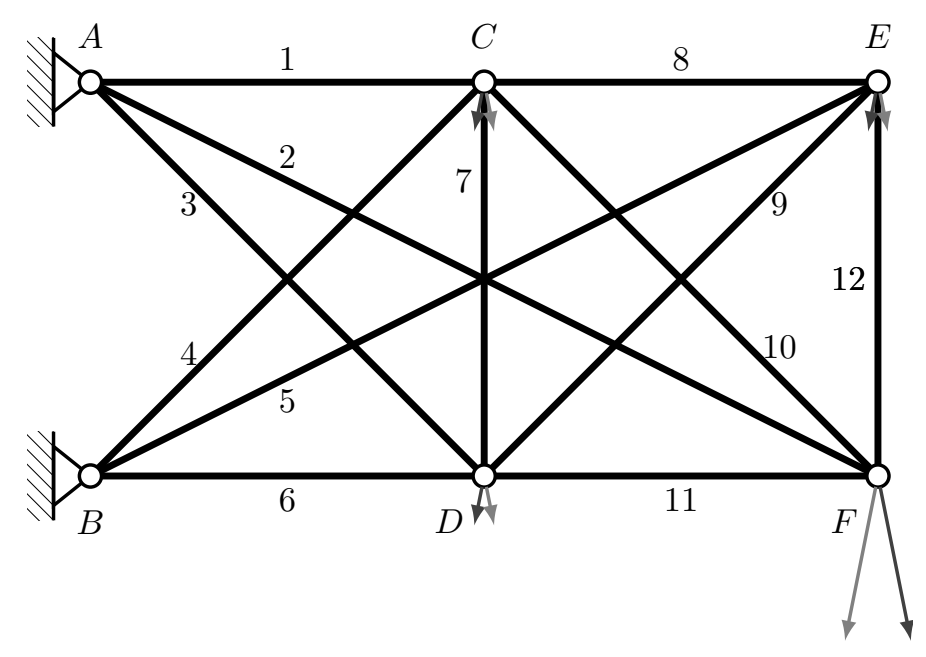

Fig. 3: Groundstructure of truss (drawn not to scale)

The optimal trusses without bar failures and without as well as with a maximum of two active bars are given in Fig. 4. Already in the case without active bars, all but one of the used bars are of the smallest possible size. With active buckling control, this bar can also be reduced to the smallest size, since the actuator can ensure the buckling constraints even with the smaller cross-sectional area, while a second active bar does not help to reduce the total number or volume of bars to further improve the objective value.

The results for $k=1$ worst-case failure are given in Fig. 5. In this case, two bars can be decreased in size through the placement of active bars. An interesting observation is that one of the active bars replaces Bar 3, which originally is under tension for both load scenarios. Therefore, this active bar will not have any influence in the original constraints without failures, since it only influences the (negative) lower bound of a positive variable. After failure of Bar 6, however, Bar 3 is severely under compression in both load scenarios and the buckling control is necessary to satisfy the lower bound of the buckling constraint for this bar. Therefore, this example shows that it is actually necessary to specifically investigate buckling constraints for the different failure scenarios, and it is not sufficient to only consider bars under compression before failures and replace those with bar forces closest to the critical buckling force by active bars.

For $k=2$ worst-case failures, the results are given in Fig. 6. Here both active bars again replace bars originally under compression to be able to reduce their sizes to smaller cross-sectional areas. Like in the previous cases, the general structure of the truss stays the same and just single bars are replaced 
by smaller active bars, but this, of course, does not have to be the case for all examples, and it can also happen that completely different structures are possible, which would not satisfy the buckling constraints before.

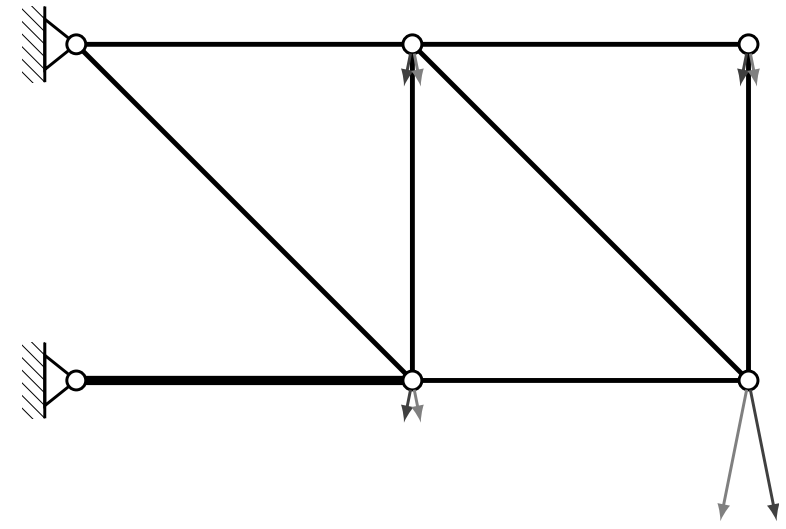

(a)

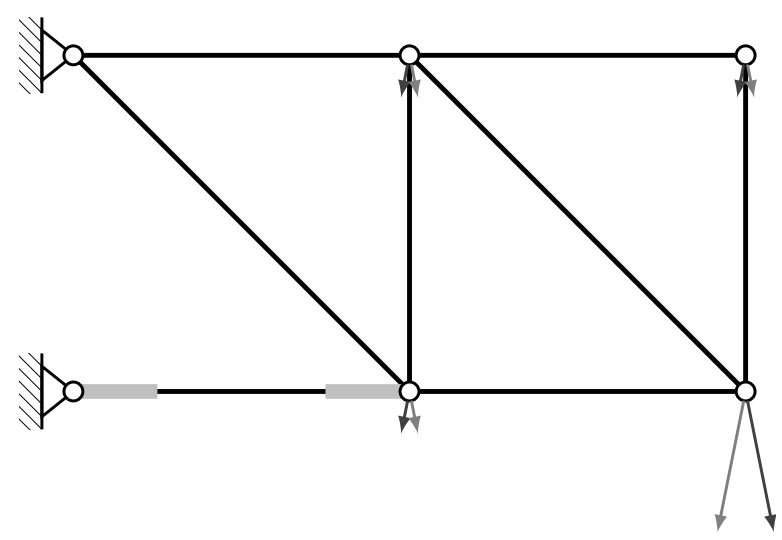

(b)

Fig. 4: Optimized truss for $k=0$ bar failures and (a) $r=0$ or (b) $r=2$ active bars

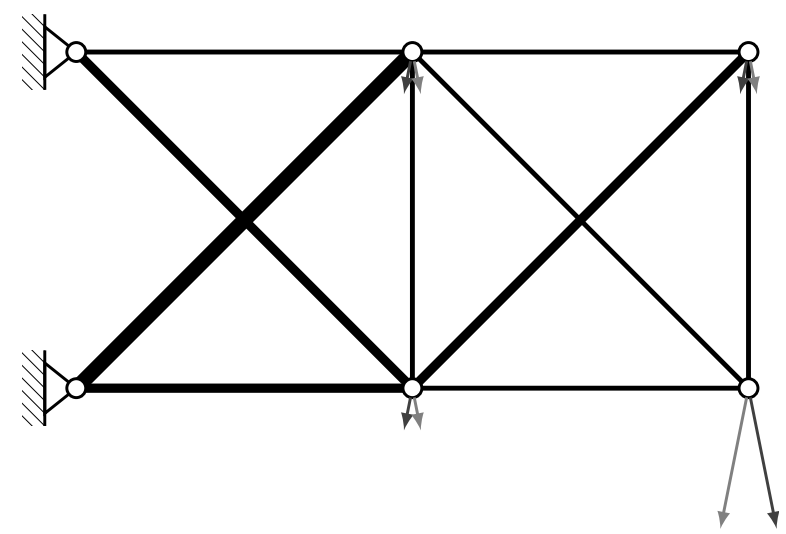

(a)

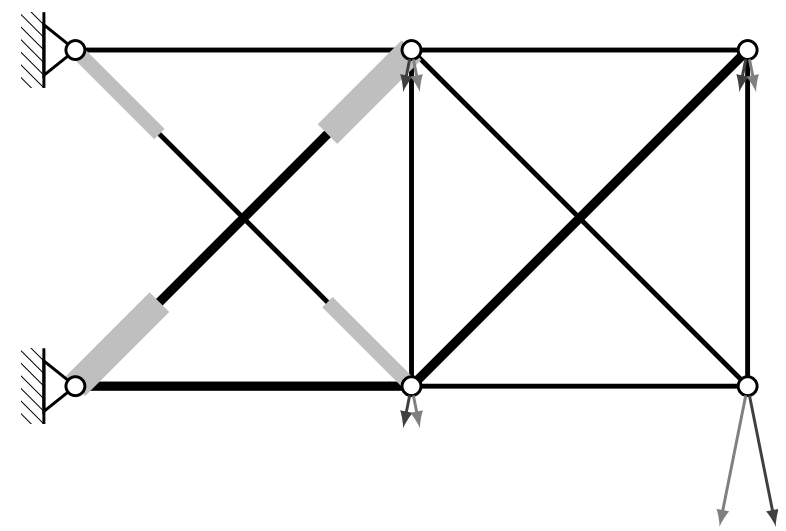

(b)

Fig. 5: Optimized truss for $k=1$ bar failures and (a) $r=0$ or (b) $r=2$ active bars

A comparison of the objective values for the different combinations of failure scenarios and number of active bars is given in Table 1(a). The first thing to be observed is that the volume increases significantly when including bar failures. Increases by more than a factor of two can also happen, since it may be necessary to have multiple thicker bars at different positions to compensate for the loss of one bar. With active bars, we always get an improvement in the objective value, but the exact factor may depend on the specific choice of possible cross-sectional areas, since we were usually able to downsize by one type when replacing a bar by an active one.

Looking at the solving times in Table 1(b), we also see a significant increase when enlarging the set of failure scenarios. For $k=2$, even for this small example, we already get a solving time of almost 900 seconds, which is 100 times more than without bar failures. But in this case we have an MISDP with almost 16000 variables, 60 of which are binary, with 132 SDP-constraints of size 9, and 19000 linear and 13000 indicator constraints. For the active bars, the influence on the solving time is much smaller, with only $m$ additional binary variables, one per bar, and $m+1$ additional linear constraints. 
In our experiments we even got a slight decrease in solving time in two of the three cases, although this should be attributed to performance variability and one should still expect a slight increase in solving times, but on a much smaller scale than for the inclusion of failure scenarios into the model.

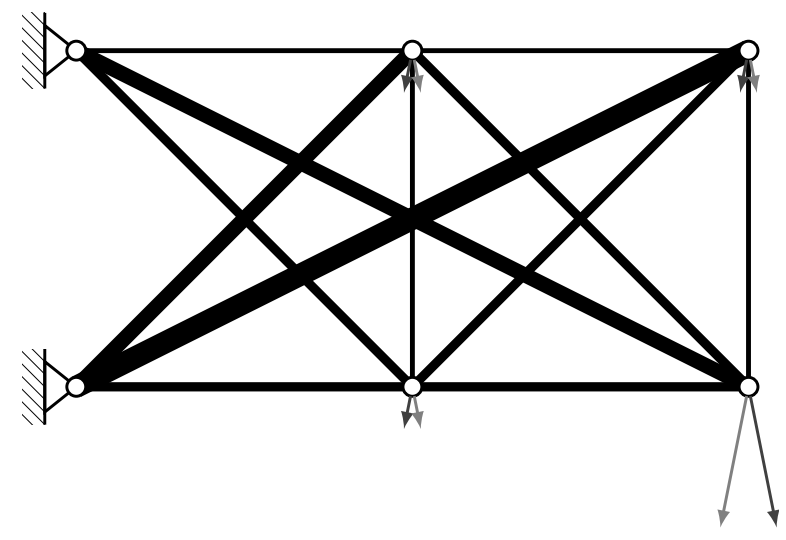

(a)

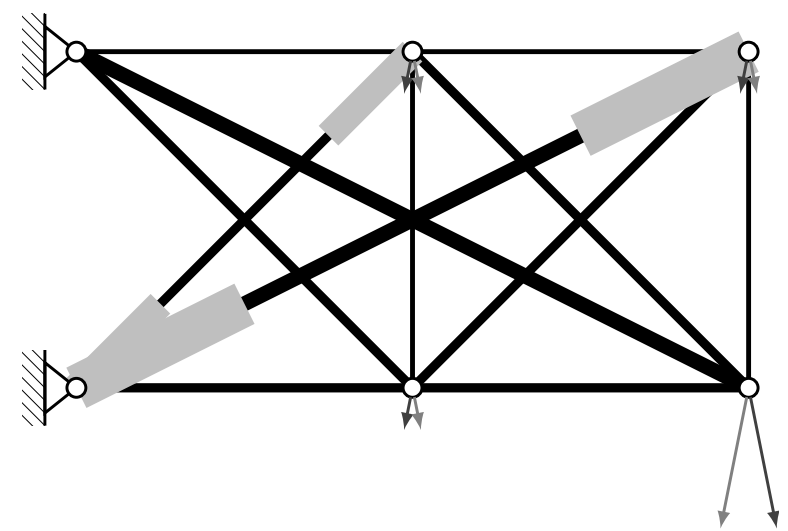

(b)

Fig. 6: Optimized truss for $k=2$ bar failures and (a) $r=0$ or (b) $r=2$ active bars

\section{Conclusion \& Outlook}

Preventing the buckling of bars is an important task in engineering applications. Instead of oversizing each bar in a truss structure, it seems more promising to use active buckling control. We have seen that bars with actuators for buckling control can increase the maximum bearable load of a single bar by up to $20 \%$. In order to use these active bars as purposefully as possible, we developed a mixedinteger semidefinite model to optimally place these active elements within truss structures even in the presence of uncertain loads and bar failures. Based on a simple truss structure with six nodes, we demonstrated how much influence the optimal placement of active bars can have.

One challenge, which could be observed in our numerical results, however, is that the MISDPs tend to get quite large even for relatively small trusses. For a problem with only six nodes, twelve bars and a maximum of two bar failures, problems with up to 16000 variables and over 30000 constraints arise, which already take quite long to solve. For being able to solve problems of more practically relevant size, these numbers would have to be decreased to find optimal solutions in acceptable times. One possibility to reduce the solving times would be to dynamically generate the additional constraints and variables necessary for the different failure scenarios. In this case, one would solve generation problems to find critical failure scenarios to add to the set of constraints (and bar forces) of the MISDP until no more critical failure scenarios can be generated. Since the resulting generation problems would be non-convex mixed-integer quadratically-constrained quadratic problems, however, one would still need to find efficient ways to solve these auxiliary problems.

Table 1: (a) Objective values for the volume $\left[10^{5} \mathrm{~mm}^{3}\right]$ and (b) solving times $[\mathrm{s}]$ for the different combinations of failure scenarios and maximal number of active bars

(a)

\begin{tabular}{l|rrr}
\hline Volume & $k=0$ & $k=1$ & $k=2$ \\
\hline$r=0$ & 1.95 & 3.61 & 7.45 \\
$r=2$ & 1.77 & 3.02 & 6.46 \\
\hline
\end{tabular}

(b)

\begin{tabular}{r|rrr}
\hline Time & $k=0$ & $k=1$ & $k=2$ \\
\hline$r=0$ & 7.47 & 52.26 & 890.23 \\
$r=2$ & 6.64 & 86.49 & 581.77 \\
\hline
\end{tabular}




\section{Acknowledgement}

The authors would like to thank the German Research Foundation (DFG) for funding this research within the Collaborative Research Center 805.

\section{References}

[1] M. P. Bendsøe and O. Sigmund, Topology Optimization: Theory, Methods and Applications. Berlin and Heidelberg: Springer, 2003.

[2] A. Ben-Tal, F. Jarre, M. Kočvara, A. Nemirovski, and J. Zowe, "Optimal design of trusses under a nonconvex global buckling constraint," Optimization and Engineering, vol. 1, pp. 189-213, 2000 .

[3] A. Ben-Tal, L. E. Ghaoui, and A. Nemirovski, Robust Optimization. Princeton University Press, 2009.

[4] P. Sun, J. Arora, and E. Haug Jr., "Fail-safe optimal design of structures," Engineering Optimization, vol. 2, pp. 43-53, 1976.

[5] M. Jansen, G. Lombaert, M. Schevenels, and O. Sigmund, "Topology optimization of fail-safe structures using a simplified local damage model," Structural and Multidisciplinary Optimization, vol. 49, no. 4, pp. 657-666, 2014.

[6] M. Zhou and R. Fleury, "Fail-safe topology optimization," Structural and Multidisciplinary Optimization, vol. 54, no. 5, pp. 1225-1243, 2016.

[7] D. P. Mohr, I. Stein, T. Matzies, and C. A. Knapek, "Redundant robust topology optimization of truss," Optimization and Engineering, vol. 15, no. 4, pp. 945-972, 2014.

[8] Y. Kanno, "Redundancy optimization of finite-dimensional structures: Concept and derivativefree algorithm," Journal of Structural Engineering, vol. 143, no. 1, 2017.

[9] D. Gross, W. Hauger, J. Schröder, and W. A. Wall, Technische Mechanik 2: Elastostatik. Berlin, Heidelberg: Springer, 2007.

[10] S. P. Timoshenko and J. M. Gere, Theory of elastic stability. New York: McGraw-Hill, 1961.

[11] M. Schäffner, B. Götz, and R. Platz, "Active buckling control of a beam-column with circular cross-section using piezo-elastic supports and integral lqr control," Smart Materials and Structures, vol. 25 , no. 6 , pp. 1-10, 2016.

[12] M. Schäffner and R. Platz, "Gain-scheduled H1 buckling control of a circular beam-column subject to time-varying axial loads," Smart Materials and Structures, 2018. to appear.

[13] A. Ben-Tal and A. Nemirovski, "Robust truss topology design via semidefinite programming," SIAM Journal on Optimization, vol. 7, no. 4, pp. 991-1016, 1997.

[14] M. Kočvara, “Truss topology design with integer variables made easy,” tech. rep., Optimization Online, 2010.

[15] S. Mars, Mixed-Integer Semidefinite Programming with an Application to Truss Topology Design. PhD thesis, FAU Erlangen-Nürnberg, 2013. 
[16] T. Gally, M. E. Pfetsch, and S. Ulbrich, "A framework for solving mixed-integer semidefinite programs," Optimization Methods and Software, vol. 33, no. 3, pp. 594-632, 2018.

[17] A. Gleixner, L. Eifler, T. Gally, G. Gamrath, P. Gemander, R. L. Gottwald, G. Hendel, C. Hojny, T. Koch, M. Miltenberger, B. Müller, M. E. Pfetsch, C. Puchert, D. Rehfeldt, F. Schlösser, F. Serrano, Y. Shinano, J. M. Viernickel, S. Vigerske, D. Weninger, J. T. Witt, and J. Witzig, "The SCIP optimization suite 5.0," Tech. Rep. 17-61, ZIB, 2017.

[18] MOSEK ApS, The MOSEK C optimizer API manual Version 8.1 (Revision 25), 2017. 